\title{
Europe's satellite for science
}

\author{
Geos, the ESA's purely scientific satellite, is launched \\ this week. Stuart Sharrock, now in Florida, reports
}

Earth Explorer). Geos is scheduled to be launched on 20 April and ISEE on 14 October; as the centrepiece of the IMS, Geos has been designated the reference spacecraft for the study and will coordinate with ground-based, balloon, aircraft and rocket programmes.

\section{The magnetosphere}

A solar wind of protons and electrons blows continuously through the solar system at some $400 \mathrm{~km} \mathrm{~s}^{-1}$ and, according to Alfven's 'frozen-in' theorem, drags with it the Sun's magnetic field. The earth's dipolar magnetic field and the ionospheric plasma are similarly frozen together. These two magnetic fields cannot mix and so the earth's field lies in a closed cavity in the plasma stream from the Sun and presents a tiny obstacle to the blast of the solar wind. This magnetic bubble is the magnetosphere; it is deformed from a dipolar configuration by the solar wind and the size of the magnetospheric cavity changes in response to the strength of the wind. At typically 10 earth radii the hot plasma from the Sun is deflected and streams around the earth's protecting magnetic bottle; under certain conditions however the bottle becomes open to the solar wind -with drastic consequences. Within the magnetosphere there are numerous plasma regions which respond to changes in the solar wind and which interact with the ionosphere and the atmosphere.

Observations with a single satellite can never distinguish unambiguously between spatial and temporal variations; two satellites orbiting together with a known separation are needed and this is one of the aims of the ISEE mission. Geos will sample the different regions of the magnetosphere, measure minute variations in all the particle and wave parameters without interference from the satellite itself, monitor conditions continuously over many months, and yet be capable of responding to changing situations in minutes, and explore the coupling between the magnetosphere and the ionosphere by enabling correlated satellite and ground-based measurements. These requirements specify a satellite with an orbit, construction and performance an order of magni-

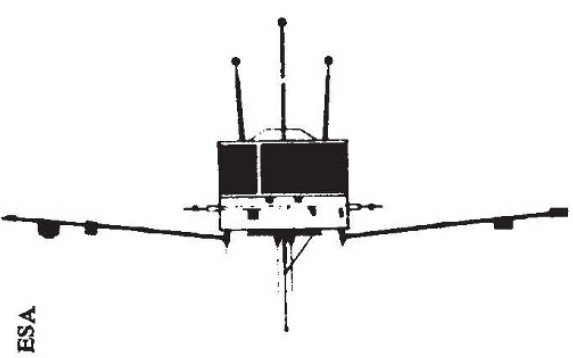

tude more complex and sophisticated than any previous ESA satellite and with a control and ground support system more ambitious than has so far been attempted by NASA with unmanned missions.

\section{The Geos satellite}

Built by a consortium of European industrial contractors headed by the British Aircraft Corporation, Bristol, England, Geos will be placed into a geostationary orbit $36,000 \mathrm{~km}$ above the equator. The orbit covers a region of the magnetosphere where many of the dynamic processes responsible for magnetic and ionospheric disturbances are believed to develop; the satellite will be positioned at longitudes where the magnetic field lines are conjugated to both auroral zones (that is, particles passing along the field lines through the satellite will arrive at the aurora), allowing correlated measurements to be made from ground stations and rocket-borne instruments.

As the spatial variations seen by Geos will be small, the time and space ambiguities will be minimal and short-lived transient phenomena can be studied. Sampling of diffenent regions of the magnetosphere will be automatic as Geos alternates between the dayside and the night-side, but the boundaries of the different magnetospheric regions are not fixed in space and will at times move across the geostationary orbit under the influence of the solar wind; phenomena associated with such boundary crossings are of particular interest.

Once in orbit, Geos will be permanently in view of a purpose-built tracking and data recovery ground station near Darmstadt in West Germany (also to be used for Meteosat). The continuous link between the satellite and the ground allows for high data rates, which are essential for some experiments, and means that real time modification of experiments becomes possible. The capability of closed loop control of instrument modes is crucial and was laid down as a ground rule for the mission in the early planning stages.

The problem with many satellite experiments is that effects caused by the spacecraft itself can often mask or destroy the phenomena of interest. Spacecraft charging is well known-
ESA venture comprising three satellites code-named ISEE (International Sun 
potentials of up to $10 \mathrm{kV}$ have been reported from the ATS satellites during eclipses-and can have disastrous effects on experiments to measure low energy particle fluxes. To minimise this problem the whole of the outer skin of Geos has been made conducting, thus ensuring that the surface is an equipotential. There is a complex system of eight booms which deploy in orbit to take the sensitive detectors up to $20 \mathrm{~m}$ away from the disturbing influence of the satellite body; this is the most complex boom system attempted for any European satellite. The experiments mounted on Geos involve extremely sensitive measurements (for example the detection of magnetic variations of a thousand millionth of the strength of the earth's magnetic field) and this has meant an extensive programme to minimise electromagnetic interference caused by equipment on the spacecraft.

\section{The experiments}

The achievement of providing a magnetospheric laboratory free from interference, accessible in real time and controllable from the ground is matched by the scope of the experiments comprising the payload. To understand the magnetosphere's structure and the wave-particle interactions that occur, it is essential to make sensitive measurements of all relevant parameters simultaneously. For the first time this is being done and data on every quantity of interest are being collected: four experiments will measure particle fluxes and their variation with energy and direction, covering a range from thermal energies to hundreds of $\mathrm{keV}$, and three experiments will measure electromagnetic waves over a frequency range $0-77 \mathrm{kHz}$ for electric fields and $0-20 \mathrm{kHz}$ for magnetic fields. DC electric and magnetic fields will be monitored with extreme sensitivity. The most important measurements will be duplicated by independent experiments.

A mission this complex cannot hope to satisfy all the requirements of a number of coordinated campaigns; many of the needs are mutually exclusive and inevitably compromises must be made. The coordination of activities is crucial to the success of the mission and has been an important factor in its planning. Areas in which the standard configuration is not ideal can hopefully be covered by special arrangements; the project has the inherent flexibility to allow this.

As an example, a polar expedition to a ground station in the Arctic could be unhappy about the orientation of Geos. The expedition will want to correlate its measurements with particle fluxes coming from the south at the position of Geos but this is only possible during the northern winter. The directional coverage for particles is not isotropic and the orientation of Geos is governed by the nequirement that the radial booms do not shadow the solar cells. Inversion of the satellite -a delicate operation-would have to be arranged but it would interfere with the wave experiments and expeditions to the Antarctic would not be too happy. Other ground stations would no doubt have conflicting requirements. Rocket campaigns, for example, are costly and can only be conducted at certain times, and these are sure to be the times when interesting transient effects are manifesting themselves and necessitating an immediate change in plan. In any case there is only a limited supply of fuel so manoeuvres are rationed. The coordinating committee will have a tough time but the number of demands made on the mission is a measure of its flexibility and importance; the number of satisfied customers is likely to be very high. Crucial to this flexibility is the provision of extensive computing facilities at the ground station where the data are collected.

\section{Coping with data}

Most of the time nothing exciting will happen (the magnetosphere can remain stable for long periods) and it is unwise to accumulate vast quantities of data throughout the mission. Filling a magnetic tape every 45 minutes, Geos would generate 26,000 tapes over a two year period of which only about $10 \%$ would be worth keeping. The solution is to recycle the magnetic tapes every three months; there is an extensive data distribution system (it includes daily summaries of all Geos data) to enable a communal decision on which data are to be retained. Not only does Geos have the ability to respond quickly to changes in the magnetospheric system, it also imposes a requirement on the experimenters to respond to their data within a reasonable time period-a valuable constraint that could be more widely adopted.

Originally planned for August 1976, the launch of Geos has been delayed until April 1977 mainly because of late delivery of components. The satellite will be launched by a NASA Delta launch vehicle from the Kennedy Space Center in Florida. The original flight plan has been continually modified to take account of the magnetic conjugacies between a given longitudinal position of the satellite and the positions of available ground stations and rocket campaigns. The current plan is to station it initially at a longitude of $0^{\circ}$, where a double magnetic conjugacy exists between Iceland in the northern hemisphere and the Syowa base in the southern hemisphere. In about August this year Greos will be drifted eastwards to arrive at about $35^{\circ} \mathrm{E}$ by October 1977; this is a position ideally suited for correlated measurements with the extensive array of ground-based stations and rocket ranges in Northern Scandinavia. In mid-1978 the satellite could be thrust back to the $0^{\circ}$ position again, further movements being a function of the status of polar expeditions, rocket campaigns, ground stations, the amount of fuel remaining, the consensus of opinion and the tempers of the Coordinating Committee.

After lift-off it will take about a month to get Geos into its correct orbit with the extensive boom system correctly deployed, another month to check out the experimental payload; by then hopefully it will be fully operational and the IMS can start in earnest. The taxpayers of Europe have made a wise investment in Geos-if NASA gets it up there.

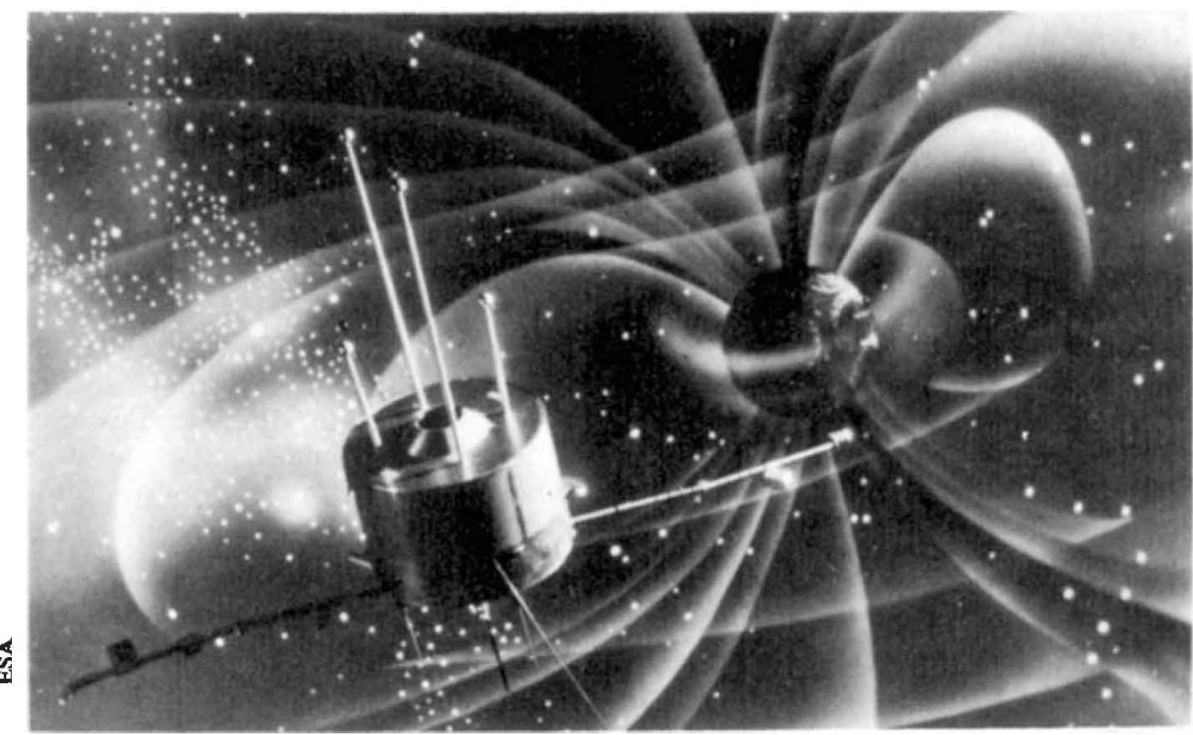

An artist's impression of Geos in the magnetosphere 